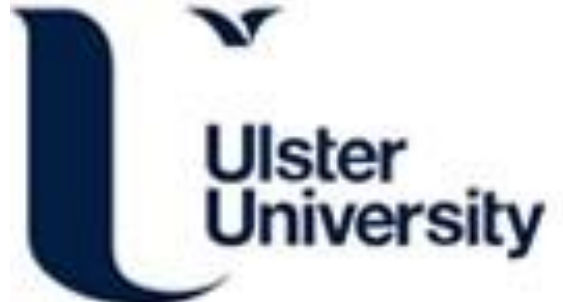

\section{Multimorbidity among persons aged 25-64 years: a population-based study of social determinants and all-cause mortality.}

Ferry, F. R., Rosato, M., Curran, E., O'Reilly, D., \& Leavey, G. (2020). Multimorbidity among persons aged 25-64 years: a population-based study of social determinants and all-cause mortality. Journal of Public Health. https://doi.org/10.1093/pubmed/fdaa209

Link to publication record in Ulster University Research Portal

Published in:

Journal of Public Health

Publication Status:

Published online: 22/12/2020

DOI:

10.1093/pubmed/fdaa209

Document Version

Publisher's PDF, also known as Version of record

\section{General rights}

Copyright for the publications made accessible via Ulster University's Research Portal is retained by the author(s) and / or other copyright owners and it is a condition of accessing these publications that users recognise and abide by the legal requirements associated with these rights.

\section{Take down policy}

The Research Portal is Ulster University's institutional repository that provides access to Ulster's research outputs. Every effort has been made to ensure that content in the Research Portal does not infringe any person's rights, or applicable UK laws. If you discover content in the Research Portal that you believe breaches copyright or violates any law, please contact pure-support@ulster.ac.uk. 


\title{
Multimorbidity among persons aged 25-64 years: a population-based study of social determinants and all-cause mortality
}

\author{
F.R. Ferry ${ }^{1}$, M.G. Rosato' ${ }^{1}$ E.J. Curran ${ }^{1}$, D. O'Reilly ${ }^{2}$, G. Leavey ${ }^{1}$ \\ ${ }^{1}$ Bamford Centre for Mental Health and Wellbeing, Room H253, School of Psychology, Ulster University, Coleraine BT52 1SA, Northern Ireland \\ ${ }^{2}$ Centre for Public Health, Block A Royal Victoria Hospital, Queen's University Belfast, Belfast BT12 6BA, Northern Ireland \\ Address correspondence to F.R. Ferry, E-mail: f.ferry@ulster.ac.uk
}

\section{ABSTRACT}

Background Despite increasing multimorbidity across the lifespan, little is known about the co-occurrence of conditions and risk factors among younger adults. This population-based study examines multimorbidity, social determinants and associated mortality among younger and middle-age adults.

Method Analysis was based on the Northern Ireland population aged 25-64 years enumerated in the 2011 Census $(n=878345)$, with all-cause mortality follow-up to 2014 (8659 deaths). Logistic regression was used to examine social determinants and Cox proportional hazards models in the analysis of associated mortality.

Results Prevalence of multimorbidity was $13.7 \%$ in females and $12.7 \%$ in males. There was a strong association between multimorbidity that included mental/cognitive illness and deprivation. Among those never married, multimorbid physical conditions were less likely [relative risk ratios $(\mathrm{RRR})=0.92: 95 \%$ confidence interval $(\mathrm{Cl})=0.88,0.95$ for males; and $\mathrm{RRR}=0.90: 0.87,0.94$ for females]. Rurality was associated with lower physical multimorbidity (RRR $=0.92: 0.89,0.95)$ but higher mental/cognitive multimorbidity (RRR $=1.35: 1.12,1.64)$ among females. All multimorbid categories were associated with elevated risk of mortality.

Conclusion The health and economic challenges created by multimorbidity should be addressed further 'upstream'. Future multimorbidity research should include younger adults to inform the development of preventative interventions and align health and social care services more closely with patients' needs.

Keywords epidemiology, morbidity and mortality, social determinants

\section{Introduction}

Life expectancy and improved childhood survival have increased the population at risk of living with both chronic conditions and persisting multimorbidity. ${ }^{1}$ While multimorbidity affects around a third of the general population at any time, ${ }^{2}$ estimates vary substantially across settings. ${ }^{3-8}$ Moreover, the economic costs of polypharmacy and fragmented care are significant. 9,10 Despite such problems, care management remains mainly focused on single conditions. ${ }^{1}$

While there is an urgent need to develop policies and strategies to more effectively identify, treat and manage multimorbidity across the lifespan, ${ }^{10}$ we know little about the interrelationships between different conditions, co-occurrence of condition combinations and the socio-demographic determinants among younger and adults, who have been shown to have higher levels of multimorbidity in absolute terms. ${ }^{5}$ Additionally, the co-ordination of care across mental and physical health conditions may add to the complexity. Building on earlier evidence focusing on long-term multiple conditions and their determinants in older age, ${ }^{11}$ we sought to examine prevalence and patterns of multimorbidity, and
F.R. Ferry,
M.G. Rosato,
E.J. Curran,
D. O'Reilly,
G. Leavey, 
the association between multimorbidity and mortality in a population group aged 25-64 years.

\section{Methods}

\section{Data}

This analysis is based on the Northern Ireland Mortality Study (NIMS). ${ }^{12}$ Briefly, it is a linkage of the enumerated 2011 Census population with subsequently occurring deaths, followedup until the end of 2014, representing 3.75 years of followup mortality data (8659 deaths). Adjusted linkage match rates (allowing for the level of completeness of the enumeration) for the 3.75 years of follow-up are $2011=97.8 \%$, $2012=98.2 \%, 2013=98.4 \%$ and $2014=98.4 \%$. All data are confidential and subject to strict controls: these are held in a secure setting by the NI Statistics and Research Agency (NISRA) and are available only to accredited researchers who access de-identified data. Use of the NI Census for research was approved by the Office for Research Ethics Committees NI.

\section{Measures}

Morbidity and multimorbidity

The 2011 Census included questions on 11 health conditions that 'have lasted, or are expected to last, at least 12 months'. These included: 'deafness/partial hearing loss, blindness/partial sight loss, communication difficulty, mobility/dexterity problems, learning or intellectual difficulty, emotional or mental health conditions, long-term pain, breathing difficulties, confusion or memory loss, chronic illness and other conditions'. Estimates of morbidity were derived from self-reported 'yes/no' responses to each of the eleven conditions. A dichotomous variable was generated to identify individuals reporting multimorbid states: (i) no multimorbidity (either no morbidity or a reported single condition) and (ii) at least two of the self-reported conditions. Additionally, conditions were identified as either 'physical' (deafness, blindness, mobility difficulty, long-term pain, breathing difficulty and chronic illness) or 'mental/cognitive' (learning difficulty, mental health condition and memory loss). 'Communication difficulties and other disorders' were excluded, as it was not possible to distinguish these as having either a physical or mental base. Finally, a six-fold classification of morbidity was generated: 'none recorded; single physical condition; single mental/cognitive condition; multimorbid physical conditions; multimorbid mental/cognitive conditions; and lastly, multimorbid physical and mental/cognitive conditions'.

\section{Mortality}

All-cause mortality was determined by examining all recorded (and linked) deaths from the General Registers Office from 2011 (subsequent to the Census) to the end of 2014.

\section{Socio-demographic and socio-economic risk factors}

Individual characteristics known to be associated with morbidity were drawn from the Census, including age group (5year bands); sex; marital status (grouped as married, never married and a single separated/divorced/widowed group); and area of residence (urban, intermediate and rural). Three variables representing socio-economic circumstance (SEC) were included: (i) household car availability (grouped as two or more cars, one car, no car access); (ii) educational attainment (university level, intermediate level, no qualifications recorded) and (iii) a combination of housing tenure and rateable value of the property. Rateable value had been derived as part of an exercise by the central government in 2010 to determine the level of local residential taxes and combined with tenure to produce a meaningful eight-fold gradation: social renting; private renting; and, for owner-occupiers, five categories ranging from less than $£ 75000$ to over $£ 200000$, with an additional category comprising 'properties as yet unvalued'.

\section{Analysis}

Analysis for this study was restricted to persons aged between 25 and 64 years at Census, living in private households and normally resident in NI $(n=878$ 345). Descriptive statistics report the prevalence of each self-reported health condition, any condition and multimorbidity. Logistic and multinomial logistic regression examined the association between socio-demographic and socio-economic factors and multimorbidity, and logistic regression models report odds ratios (OR) and multinomial models report relative risk ratios (RRR). Cox proportion hazards models examined all-cause mortality. All analyses were completed using Stata version 15.0.

\section{Results}

\section{Prevalence of morbidity and multimorbidity}

The overall prevalence of 'morbidity' (any self-reported health condition) among all adults aged 25-64 years was $31.8 \%$ (31.4\% among males and 32.2\% among females). The three most prevalent conditions for both males and females were long-term pain $(10.4 \%$ for males, $12.1 \%$ for females), mobility problems ( $9.7 \%$ for males, $10.9 \%$ for 
females) and mental health problems (7.3\% for males and $10.1 \%$ for females). 'Multimorbidity' (presence of two or more conditions) was reported by $13.2 \%$ of all persons and was more prevalent in females $(13.7 \%)$ compared to males $(12.7 \%)$.

\section{Socio-demographic and socio-economic associations with multimorbidity}

Table 1 examines multimorbidity per se in comparison with a reference category comprising people reporting either no morbidity or a single morbid condition. ORs show the likelihood of multimorbidity associated with socio-demographic and socio-economic characteristics. Among all persons, multimorbidity was higher in females when compared with males $[\mathrm{OR}=1.09: 95 \%$ confidence interval $(\mathrm{CI})=1.08,1.11]$ and increasing with age $(\mathrm{OR}=8.40: 8.11,8.71$ for those aged $60-$ 64). There was a strong protective effect associated with being married, and those living in intermediate and rural settings (when compared to their urban peers). Housing tenure/property value as a marker for SEC showed that those in social rented accommodation were at higher risk of multimorbidity $(\mathrm{OR}=3.26: 3.15,3.38)$ compared to people living in the most expensive owner-occupied housing. These patterns are largely reflected in analysis stratified by sex, with the exception of area of residence, which was not associated with multimorbidity for males.

\section{Socio-demographic and socio-economic associations with morbidity categories}

As previously indicated, individuals were assigned to a mutually exclusive morbidity category based on the number and type of self-reported health conditions. Tables 2 and 3 show, in multinomial models stratified by sex, the likelihoods associated with the selected risk factors and the morbidity/multimorbidity outcomes. RRR are presented and are based on fully adjusted models. Minimally adjusted model results are available on request.

\section{Age}

Generally, for both males and females, relative risks associated with physical morbidities increased uniformly by age (for example, for multimorbid physical outcomes RRR $=26.72$ : 95\% CI $=24.40,29.26$; and $\mathrm{RRR}=19.47: 17.94,21.13$ for males and females aged 60-64, respectively). Outcomes comprising mental/cognitive morbidities only are distributed more evenly through the age groups and peak in middle-age before declining (for example, for multimorbid mental/cognitive outcomes $R R R=1.95: 1.52,2.48$ for males aged 55-59; and $R R R=2.27: 1.74,2.96$ for females aged 45-49).

\section{Marital status}

When compared to their currently married peers, those widowed/separated or divorced had increased risk across all morbidity outcomes, recording elevated likelihoods of multimorbid mental/cognitive conditions $(\mathrm{RRR}=3.39$ : $95 \% \mathrm{CI}=2.79,4.12$ for males and $\mathrm{RRR}=2.08: 1.70,2.53$ for females). While those never married recorded elevated odds associated with multiple mental/cognitive conditions and generally show higher likelihoods over all morbidity outcomes, this group shows some advantage with respect to physical health, with both males and females less likely to report multimorbid physical conditions $(\mathrm{RRR}=0.92: 0.88,0.95$ for males and $\mathrm{RRR}=0.90: 0.87,0.94$ for females).

\section{Socio-economic circumstance}

The relative risks associated with SEC reflect the standard social-class gradients associated with health outcomes. The risk of multimorbid outcomes with a mental health component is considerable among the most disadvantaged. For example, the likelihoods of combined physicalmental/cognitive morbidities for those in social rented accommodation was $\mathrm{RRR}=4.54: 4.16,4.95$ (males) and $\mathrm{RRR}=4.92: 4.55,5.32$ (females), while the risk of multiple mental/cognitive health morbidities among those with no educational qualifications was $\mathrm{RRR}=5.27: 4.37,6.36$ for males and RRR $=6.21: 5.00,7.72$ for females.

\section{Area of residence}

The analysis provides no evidence of an association between area of residence for males. However, for females, we found rurality associated with an increased likelihood of multimorbid mental/cognitive outcomes $(\mathrm{RRR}=1.35$ : 1.12, 1.64) and decreased likelihood of multimorbid physical outcomes $(\mathrm{RRR}=0.92: 0.89,0.95)$. Additionally, multimorbid physical outcomes and multimorbid physical-mental/cognitive outcomes were less likely among females living in intermediate settings $(\mathrm{RRR}=0.93: 0.91,0.96$ and $\mathrm{RRR}=0.93: 0.90,0.96$, respectively).

\section{All-cause mortality and morbidity}

Table 4 shows the mortality risks associated with each of the multimorbidity classifications: overall there were 8659 deaths during the follow-up period. Results show increased mortality across all five morbidity categories in the follow-up period compared to those with no morbidity, with highest risk associated with the three 'multimorbidity' categories for both males and females. Likelihoods were notably elevated for both multimorbidity categories with a physical health component: 
Table 1 Socio-demographic and socio-economic characteristics associations with multimorbidity among persons aged 25-64

\begin{tabular}{|c|c|c|c|c|}
\hline & & All persons aged 25-64 & Males aged 25-64 & Females aged 25-64 \\
\hline & $\%$ of group & Fully adjusted ${ }^{a}$ OR $(95 \%$ Cl) & Fully adjusted ${ }^{a}$ OR $(95 \% \mathrm{CI})$ & Fully adjusted ${ }^{a}$ OR $(95 \% \mathrm{CI})$ \\
\hline \multicolumn{5}{|l|}{ Age } \\
\hline $25-29$ & 4.31 & 1.00 & 1.00 & 1.00 \\
\hline $30-34$ & 5.19 & $1.37(1.32,1.43)^{* * *}$ & $1.31(1.23,1.39)^{* * *}$ & $1.43(1.35,1.51)^{* * *}$ \\
\hline $35-39$ & 7.36 & $2.04(1.97,2.12)^{* * *}$ & $1.90(1.80,2.01)^{* * *}$ & $2.16(2.06,2.27)^{* * *}$ \\
\hline $40-44$ & 11.32 & $2.91(2.81,3.02)^{* * *}$ & $2.65(2.52,2.80)^{* * *}$ & $3.12(2.97,3.27)^{* * *}$ \\
\hline $45-49$ & 14.77 & $3.89(3.76,4.03)^{* * *}$ & $3.61(3.43,3.80)^{* * *}$ & $4.11(3.91,4.31)^{* * *}$ \\
\hline $50-54$ & 17.22 & $5.21(5.03,5.40)^{* * *}$ & $4.97(4.72,5.23)^{* * *}$ & $5.38(5.12,5.65)^{* * *}$ \\
\hline $55-59$ & 18.39 & $6.90(6.66,7.15)^{* * *}$ & $6.85(6.51,7.21)^{* * *}$ & $6.86(6.53,7.20)^{* * *}$ \\
\hline $60-64$ & 21.44 & $8.40(8.11,8.71)^{* * *}$ & $8.99(8.54,9.47)^{* * *}$ & $7.80(7.42,8.20)^{* * *}$ \\
\hline \multicolumn{5}{|l|}{ Sex } \\
\hline Male & 12.66 & 1.00 & & \\
\hline Female & 13.67 & $1.09(1.08,1.11)^{* * *}$ & & \\
\hline \multicolumn{5}{|l|}{ Marital status } \\
\hline Married & 11.23 & 1.00 & 1.00 & 1.00 \\
\hline Never married & 11.32 & $1.21(1.18,1.23)^{* * *}$ & $1.24(1.21,1.28)^{* * *}$ & $1.17(1.14,1.20)^{* * *}$ \\
\hline Widowed/Separated/Divorced & 24.46 & $1.28(1.25,1.30)^{* * *}$ & $1.26(1.23,1.30)^{* * *}$ & $1.29(1.26,1.32)^{* * *}$ \\
\hline \multicolumn{5}{|l|}{ Tenure/property value ${ }^{b}$} \\
\hline f200+ & 6.56 & 1.00 & 1.00 & 1.00 \\
\hline f150-f199 & 8.62 & $1.25(1.21,1.30)^{* * *}$ & $1.24(1.18,1.30)^{* * *}$ & $1.26(1.21,1.33)^{* * *}$ \\
\hline f100-f149 & 10.77 & $1.46(1.41,1.50)^{* * *}$ & $1.36(1.30,1.42)^{* * *}$ & $1.55(1.48,1.62)^{* * *}$ \\
\hline f75-f99 & 13.31 & $1.61(1.56,1.67)^{* * *}$ & $1.50(1.43,1.58)^{* * *}$ & $1.72(1.64,1.80)^{* * *}$ \\
\hline$<\mathrm{f75}$ & 14.77 & $1.57(1.52,1.63)^{* * *}$ & $1.46(1.39,1.54)^{* * *}$ & $1.68(1.59,1.76)^{* * *}$ \\
\hline Privately rented & 13.98 & $2.07(2.00,2.14)^{* * *}$ & $1.89(1.80,1.99)^{* * *}$ & $2.24(2.13,2.34)^{* * *}$ \\
\hline Social renting & 30.53 & $3.26(3.15,3.38)^{* * *}$ & $3.16(3.00,3.32)^{* * *}$ & $3.34(3.18,3.50)^{* * *}$ \\
\hline Value not assigned & 6.81 & $1.18(1.13,1.24)^{* * *}$ & $1.11(1.04,1.18)^{* *}$ & $1.26(1.19,1.34)^{* * *}$ \\
\hline \multicolumn{5}{|l|}{ Household car access } \\
\hline Two or more & 8.08 & 1.00 & 1.00 & 1.00 \\
\hline One & 15.83 & $1.47(1.45,1.50)^{* * *}$ & $1.56(1.52,1.60)^{* * *}$ & $1.40(1.37,1.43)^{* * *}$ \\
\hline None & 26.08 & $1.84(1.79,1.88)^{* * *}$ & $2.02(1.95,2.09)^{* * *}$ & $1.69(1.64,1.75)^{* * *}$ \\
\hline \multicolumn{5}{|l|}{ Area } \\
\hline Urban & 14.59 & 1.00 & 1.00 & 1.00 \\
\hline Intermediate & 13.66 & $0.97(0.95,0.98)^{* * *}$ & $1.00(0.98,1.02)$ & $0.94(0.92,0.96)^{* * *}$ \\
\hline Rural & 10.67 & $0.97(0.95,0.99)^{* *}$ & $0.98(0.96,1.01)$ & $0.96(0.93,0.98)^{* *}$ \\
\hline \multicolumn{5}{|l|}{ Education } \\
\hline Degree & 6.64 & 1.00 & 1.00 & 1.00 \\
\hline Intermediate level & 10.89 & $1.49(1.46,1.52)^{* * *}$ & $1.53(1.49,1.58)^{* * *}$ & $1.46(1.42,1.49)^{* * *}$ \\
\hline No qualifications & 27.10 & $2.43(2.39,2.48)^{* * *}$ & $2.48(2.41,2.55)^{* * *}$ & $2.42(2.35,2.48)^{* * *}$ \\
\hline
\end{tabular}

Findings represent OR and $95 \% \mathrm{Cls}$ from logistic regression models.

Models fully adjusted for all variables in the table. Age and sex adjusted models available on request.

Value measured in thousands of pounds sterling.

${ }^{* * *} P<0.001 ;{ }^{* *} P<0.005$.

multimorbid physical [hazard ratios $(\mathrm{HR})=3.20$ : $95 \%$ $\mathrm{CI}=2.95,3.47$ for males and $\mathrm{HR}=4.11: 3.74,4.51$ for females] and multimorbid physical-mental/cognitive $(\mathrm{HR}=2.98: 2.73,3.26$ for males and $\mathrm{HR}=3.84: 3.47,4.25)$ in fully adjusted models.

\section{Discussion}

\section{Main finding of this study}

Our study indicates that multimorbidity is a pertinent health issue across the lifespan, echoing findings by Barnett $e t a l .^{5}$ 
Table 2 Males (25-64): socio-demographic and socio-economic variation across differing levels of multimorbidity

\begin{tabular}{|c|c|c|c|c|c|}
\hline & Single physical RRR $(95 \%$ CI) & $\begin{array}{l}\text { Single mental/cognitive RRR } \\
(95 \% \mathrm{CI})\end{array}$ & $\begin{array}{l}\text { Multimorbid physical RRR } \\
(95 \% \mathrm{CI})\end{array}$ & $\begin{array}{l}\text { Multimorbid mental/cognitive } \\
\text { RRR }(95 \% \mathrm{CI})\end{array}$ & $\begin{array}{l}\text { Multimorbid } \\
\text { physical-mental/cognitive RRR } \\
(95 \% \mathrm{CI})\end{array}$ \\
\hline \multicolumn{6}{|l|}{ Age group } \\
\hline $25-29$ & 1.00 & 1.00 & 1.00 & 1.00 & 1.00 \\
\hline $30-34$ & $1.18(1.13,1.24)^{* * *}$ & $1.33(1.25,1.43)^{* * *}$ & $1.52(1.37,1.69)^{* * *}$ & $1.69(1.39,2.05)^{* * *}$ & $1.30(1.20,1.42)^{* * *}$ \\
\hline $35-39$ & $1.48(1.41,1.54)^{* * *}$ & $1.66(1.56,1.78)^{* * *}$ & $2.61(2.37,2.89)^{* * *}$ & $1.76(1.44,2.14)^{* * *}$ & $1.99(1.84,2.15)^{* * *}$ \\
\hline $40-44$ & $1.80(1.72,1.88)^{* * *}$ & $1.86(1.74,1.99)^{* * *}$ & $4.00(3.64,4.40)^{* * *}$ & $1.89(1.55,2.30)^{* * *}$ & $2.85(2.65,3.07)^{* * *}$ \\
\hline $45-49$ & $2.26(2.17,2.36)^{* * *}$ & $1.95(1.82,2.08)^{* * *}$ & $5.99(5.46,6.57)^{* * *}$ & $1.90(1.54,2.33)^{* * *}$ & $4.00(3.72,4.30)^{* * *}$ \\
\hline $50-54$ & $2.92(2.80,3.05)^{* * *}$ & $2.12(1.97,2.28)^{* * *}$ & $9.84(8.98,10.79)^{* * *}$ & $1.89(1.51,2.36)^{* * *}$ & $5.29(4.91,5.69)^{* * *}$ \\
\hline $55-59$ & $3.91(3.74,4.09)^{* * *}$ & $2.11(1.95,2.28)^{* * *}$ & $16.35(14.93,17.91)^{* * *}$ & $1.95(1.52,2.48)^{* * *}$ & $6.66(6.18,7.17)^{* * *}$ \\
\hline $60-64$ & $5.40(5.17,5.65)^{* * *}$ & $1.92(1.77,2.10)^{* * *}$ & $26.72(24.40,29.26)^{* * *}$ & $1.60(1.21,2.10)^{* *}$ & $7.58(7.02,8.17)^{* * *}$ \\
\hline \multicolumn{6}{|l|}{ Marital status } \\
\hline Married & 1.00 & 1.00 & 1.00 & 1.00 & 1.00 \\
\hline Never married & $1.04(1.02,1.07)^{* *}$ & $2.74(2.62,2.87)$ & $0.92(0.88,0.95)^{* * *}$ & $5.24(4.42 .6 .22)^{* * *}$ & $1.61(1.55,1.68)^{* * *}$ \\
\hline $\begin{array}{l}\text { Widowed/Separated/Di- } \\
\text { vorced }\end{array}$ & $1.11(1.08,1.15)^{* * *}$ & $2.00(1.90,2.12)^{* * *}$ & $1.08(1.04,1.13)^{* * *}$ & $3.39(2.79,4.12)^{* * *}$ & $1.74(1.67,1.81)^{* * *}$ \\
\hline \multicolumn{6}{|l|}{ Tenure/property value } \\
\hline$£ 200000+$ & 1.00 & 1.00 & 1.00 & 1.00 & 1.00 \\
\hline$£ 150-£ 199999$ & $1.12(1.08,1.16)$ & $1.08(0.98,1.19)$ & $1.30(1.22,1.39)^{* * *}$ & $1.12(0.74,1.72)$ & $1.30(1.18,1.43)^{* * *}$ \\
\hline$f_{100} 000-£_{, 149} 999$ & $1.15(1.11,1.19)^{* * *}$ & $1.09(1.00,1.19)$ & $1.49(1.41,1.58)^{* * *}$ & $1.00(0.69,1.46)$ & $1.47(1.35,1.60)^{* * *}$ \\
\hline$f_{75} 000-f_{f} 99999$ & $1.18(1.13,1.23)^{* * *}$ & $1.04(0.95,1.15)$ & $1.69(1.59,1.80)^{* * *}$ & $0.98(0.67,1.45)$ & $1.69(1.55,1.84)^{* * *}$ \\
\hline$<£, 75000$ & $1.22(1.16,1.27)^{* * *}$ & $0.99(0.90,1.10)$ & $1.75(1.63,1.87)^{* * *}$ & $0.89(0.60,1.33)$ & $1.57(1.44,1.72)^{* * *}$ \\
\hline Privately rented & $1.15(1.10,1.20)^{* * *}$ & $1.31(1.20,1.44)^{* * *}$ & $1.95(1.82,2.09)^{* * *}$ & $1.48(1.02,2.16)^{*}$ & $2.39(2.19,2.60)^{* * *}$ \\
\hline Social renting & $1.64(1.56,1.72)^{* * *}$ & $2.07(1.89,2.27)^{* * *}$ & $3.63(3.39,3.88) * * *$ & $2.78(1.91,4.04)^{* * *}$ & $4.54(4.16,4.95)^{* * *}$ \\
\hline No value assigned & $1.05(1.00,1.10)$ & $0.91(0.81,1.02)$ & $1.25(1.15,1.36)^{* * *}$ & $0.94(0.59,1.50)$ & $1.09(0.97,1.22)$ \\
\hline \multicolumn{6}{|l|}{ Household car access } \\
\hline Two or more & 1.00 & 1.00 & 1.00 & 1.00 & 1.00 \\
\hline One car & $1.12(1.10,1.15)^{* * *}$ & $1.56(1.49,1.64)^{* * *}$ & $1.51(1.46,1.56)^{* * *}$ & $1.95(1.64,2.33)^{* * *}$ & $1.92(1.85,2.00)^{* * *}$ \\
\hline None & $1.30(1.26,1.35)^{* * *}$ & $2.86(2.70,3.03)^{* * *}$ & $1.67(1.59,1.75)^{* * *}$ & $4.90(4.05,5.93)^{* * *}$ & $3.02(2.87,3.17)^{* * *}$ \\
\hline \multicolumn{6}{|l|}{ Area } \\
\hline Urban & 1.00 & 1.00 & 1.00 & 1.00 & 1.00 \\
\hline Intermediate & $1.00(0.98,1.03)$ & $1.03(0.99,1.07)$ & $1.02(0.98,1.05)$ & $1.03(0.92,1.16)$ & $0.99(0.95,1.02)$ \\
\hline Rural & $0.97(0.95,0.99)^{*}$ & $0.99(0.94,1.04)$ & $1.00(0.97,1.04)$ & $1.10(0.94,1.29)$ & $0.97(0.93,1.01)$ \\
\hline \multicolumn{6}{|l|}{ Education level } \\
\hline Degree or higher & 1.00 & 1.00 & 1.00 & 1.00 & 1.00 \\
\hline Intermediate level & $1.23(1.20,1.26)^{* * *}$ & $1.37(1.31,1.44)^{* * *}$ & $1.59(1.54,1.65)^{* * *}$ & $2.03(1.68,2.45)^{* * *}$ & $1.68(1.60,1.76)^{* * *}$ \\
\hline No qualifications & $.32(1.29,1.36)^{* * *}$ & $2.43(2.31,2.56)^{* * *}$ & $2.19(2.10,2.28) * * *$ & $5.27(4.37,6.36)^{* * *}$ & $3.55(3.39,3.72)^{* * *}$ \\
\hline
\end{tabular}

Results represent RRR and 95\% Cls derived from fully adjusted multinomial logistic regressions (reference group = no morbidity).

${ }^{* * *} P<0.001$; $^{* *} P<0.005$.

Consistent with results on older age adults in NI, ${ }^{11}$ we found elevated risks of premature mortality associated with all categories of multimorbidity among those aged 25-64 years. Some noteworthy findings also emerged from analysis of social determinants of multimorbidity and morbidity among younger adults. Our study found a high risk of multimorbidity with a mental/cognitive component among the most disadvantaged. While marriage was protective against multimorbidity per se, the study points to some advantage with respect to physical health among people who were never married. Females living in more rural settings were also less likely to have multimorbidity with a physical component, but more likely to have mental/cognitive multimorbidity.

\section{What is already known on this topic}

Governments and healthcare systems acknowledge the urgent need to re-orientate management of care away from traditional single condition models. However, Navickas et al. ${ }^{13}$ suggest that progress towards more sustainable care models is hampered by limited evidence on the prevalence, risks and aetiology of multimorbidity, particularly in younger age groups. ${ }^{14,15}$ Taylor et al. ${ }^{14}$ found multimorbidity prevalence of $\sim 4 \%$ in adults aged $20-39$ years and $15 \%$ at $40-59$ years compared to $39 \%$ in those aged sixty or more. McLean et al. ${ }^{15}$ found a varying profile of conditions among younger and older adults with multimorbidity more likely comprising of mixed mental and physical conditions among younger adults. More evidence is required on the types of chronic conditions that commonly occur, their risk factors and their impact on impairment, service use and mortality among younger and middle-age adults to better inform a lifespan approach to multimorbidity and allow targeting of scarce resources.

Violan et al. ${ }^{16}$ concluded that multimorbidity is consistently associated with increasing age, lower socio-economic status and female gender. A cross-sectional study, Asfar et al., ${ }^{17}$ across 28 countries supports these broad findings. 
Table 3 Females (25-64): socio-demographic and socio-economic variation across differing levels of multimorbidity

\begin{tabular}{|c|c|c|c|c|c|}
\hline & Single physical RRR $(95 \% \mathrm{CI})$ & $\begin{array}{l}\text { Single mental/cognitive RRR } \\
(95 \% \mathrm{CI})\end{array}$ & $\begin{array}{l}\text { Multimorbid physical RRR } \\
(95 \% \text { CI })\end{array}$ & $\begin{array}{l}\text { Multimorbid mental/cognitive } \\
\text { RRR }(95 \% \mathrm{CI})\end{array}$ & $\begin{array}{l}\text { Multimorbid } \\
\text { physical-mental/cognitive RRR } \\
(95 \% \mathrm{CI})\end{array}$ \\
\hline \multicolumn{6}{|l|}{ Age } \\
\hline 25-29 & 1.00 & 1.00 & 1.00 & 1.00 & 1.00 \\
\hline $30-34$ & $1.13(1.08,1.18)^{* * *}$ & $1.37(1.30,1.45)^{* * *}$ & $1.49(1.36,1.64)^{* * *}$ & $1.56(1.21,2.02)^{* *}$ & $1.50(1.40,1.62)^{* * *}$ \\
\hline $35-39$ & $1.37(1.31,1.43)^{* * *}$ & $1.74(1.65,1.84)^{* * *}$ & $2.35(2.15,2.57)^{* * *}$ & $2.23(1.73,2.86)^{* * *}$ & $2.50(2.33,2.68)^{* * *}$ \\
\hline $40-44$ & $1.65(1.58,1.72)^{* * *}$ & $1.71(1.62,1.81)^{* * *}$ & $3.77(3.47,4.11)^{* * *}$ & $2.22(1.72,2.86) * * *$ & $3.60(3.37,3.85)^{* * *}$ \\
\hline $45-49$ & $2.03(1.95,2.12)^{* * *}$ & $1.76(1.66,1.86)^{* * *}$ & $5.61(5.17,6.10)^{* * *}$ & $2.27(1.74,2.96)^{* * *}$ & $4.67(4.37,4.99)^{* * *}$ \\
\hline $50-54$ & $2.58(2.47,2.69)^{* * *}$ & $1.50(1.41,1.60)^{* * *}$ & $8.59(7.91,9.32)^{* * *}$ & $2.20(1.65,2.92)^{* * *}$ & $5.78(5.40,6.19)^{* * *}$ \\
\hline $55-59$ & $3.26(3.12,3.41)^{* * *}$ & $1.41(1.31,1.51)^{* * *}$ & $13.42(12.36,14.57)^{* * *}$ & $1.84(1.34,2.53)^{* * *}$ & $6.56(6.12,7.03)^{* * *}$ \\
\hline $60-64$ & $4.29(4.10,4.48)^{* * *}$ & $0.88(0.81,0.95)^{* *}$ & $19.47(17.94,21.13)^{* * *}$ & $1.34(0.95,1.90)$ & $5.69(5.30,6.11)^{* * *}$ \\
\hline \multicolumn{6}{|l|}{ Marital status } \\
\hline Married & 1.00 & 1.00 & 1.00 & 1.00 & 1.00 \\
\hline Never married & $1.06(1.03,1.09)^{* * *}$ & $1.55(1.49,1.61)^{* * *}$ & $0.90(0.87,0.94)^{* * *}$ & $3.22(2.66,3.90) * * *$ & $1.43(1.38,1.49)^{* * *}$ \\
\hline Widowed/Separated/Divorced & $1.07(1.05,1.10)^{* * *}$ & $1.72(1.66,1.79)^{* * *}$ & $1.06(1.02,1.09)^{* *}$ & $2.08(1.70,2.53)^{* * *}$ & $1.78(1.72,1.84)^{* * *}$ \\
\hline \multicolumn{6}{|l|}{ Tenure/property value } \\
\hline$£ 200000+$ & 1.00 & 1.00 & 1.00 & 1.00 & 1.00 \\
\hline$£$,150000-£,199999 & $1.14(1.10,1.19)^{* * *}$ & $1.24(1.14,1.34)^{* * *}$ & $1.28(1.20,1.36)^{* * *}$ & $1.30(0.80,2.11)$ & $1.38(1.27,1.49)^{* * *}$ \\
\hline$f_{100000-£, 149999}$ & $1.26(1.21,1.31)^{* * *}$ & $1.34(1.25,1.44)^{* * *}$ & $1.64(1.55,1.73)^{* * *}$ & $1.16(0.74,1.81)$ & $1.74(1.62,1.88)^{* * *}$ \\
\hline f75 000-£.99999 & $1.37(1.32,1.43)^{* * *}$ & $1.40(1.30,1.51)^{* * *}$ & $1.83(1.72,1.95)^{* * *}$ & $1.44(0.92,2.26)$ & $2.07(1.91,2.23)^{* * *}$ \\
\hline$<£ 75000$ & $1.39(1.33,1.45) * * *$ & $1.35(1.24,1.47)^{* * *}$ & $1.83(1.71,1.95)^{* * *}$ & $1.33(0.83,2.13)$ & $2.02(1.86,2.19)^{* * *}$ \\
\hline Privately rented & $1.32(1.27,1.38)^{* * *}$ & $1.68(1.55,1.81)^{* * *}$ & $2.11(1.98,2.26)^{* * *}$ & $2.01(1.29,3.14)^{* *}$ & $2.98(2.76,3.23)^{* * *}$ \\
\hline Social renting & $1.79(1.71,1.88)^{* * *}$ & $2.32(2.14,2.51)^{* * *}$ & $3.60(3.37,3.84)^{* * *}$ & $2.71(1.74,4.22)^{* * *}$ & $4.92(4.55,5.32)^{* * *}$ \\
\hline No value assigned & $1.15(1.09,1.21)^{* * *}$ & $1.15(1.04,1.26)^{* *}$ & $1.34(1.24,1.46)^{* * *}$ & $1.18(0.68,2.06)$ & $1.31(1.18,1.45)^{* * *}$ \\
\hline \multicolumn{6}{|l|}{ Household car access } \\
\hline Two or more & 1.00 & 1.00 & 1.00 & 1.00 & 1.00 \\
\hline One car & $1.14(1.11,1.16)^{* * *}$ & $1.51(1.45,1.57)^{* * *}$ & $1.38(1.33,1.42)^{* * *}$ & $1.73(1.40,2.13)^{* * *}$ & $1.63(1.57,1.68)^{* * *}$ \\
\hline None & $1.30(1.25,1.35)^{* * *}$ & $2.26(2.15,2.37)^{* * *}$ & $1.55(1.47,1.62)^{* * *}$ & $3.32(2.62,4.21)^{* * *}$ & $2.27(2.17,2.38)^{* * *}$ \\
\hline \multicolumn{6}{|l|}{ Area } \\
\hline Urban & 1.00 & 1.00 & 1.00 & 1.00 & 1.00 \\
\hline Intermediate & $1.01(0.99,1.03)$ & $1.01(0.97,1.04)$ & $0.93(0.91,0.96) * * *$ & $1.13(0.97,1.30)$ & $0.93(0.90,0.96)^{* * *}$ \\
\hline Rural & $0.98(0.96,1.00)$ & $1.04(1.00,1.09)$ & $0.92(0.89,0.95)^{* * *}$ & $1.35(1.12,1.64)^{* *}$ & $0.99(0.95,1.03)$ \\
\hline \multicolumn{6}{|l|}{ Education level } \\
\hline Degree or higher & 1.00 & 1.00 & 1.00 & 1.00 & 1.00 \\
\hline Intermediate level & $1.16(1.14,1.19)^{* * *}$ & $1.63(1.58,1.70)^{* * *}$ & $1.37(1.32,1.41)^{* * *}$ & $1.78(1.43,2.21)^{* * *}$ & $1.84(1.77,1.91)^{* * *}$ \\
\hline No qualifications & $1.40(1.36,1.44)^{* * *}$ & $2.56(2.45,2.67) * * *$ & $2.09(2.02,2.17)^{* * *}$ & $6.21(5.00,7.72)^{* * *}$ & $3.79(3.64,3.95) * * *$ \\
\hline
\end{tabular}

Results represent RRR and 95\% Cls derived from a fully adjusted multinomial logistic regression (reference group = no morbidity) ${ }^{* * *} P<0.001 i^{* *} P<0.005$

Table 4 All-cause mortality associated with categories of morbidity/multimorbidity

\begin{tabular}{|c|c|c|c|c|c|c|c|}
\hline \multirow[b]{2}{*}{ Morbidity category } & \multirow[b]{2}{*}{ Deaths (\%) } & \multicolumn{2}{|c|}{ All persons aged 25-64 } & \multicolumn{2}{|c|}{ Males aged 25-64 } & \multicolumn{2}{|c|}{ Females aged 25-64 } \\
\hline & & $\begin{array}{l}\text { Unadjusted HR } \\
(95 \% \mathrm{CI})\end{array}$ & $\begin{array}{l}\text { Fully adjusted HR } \\
(95 \% \mathrm{CI})^{\mathrm{a}}\end{array}$ & $\begin{array}{l}\text { Unadjusted HR } \\
(95 \% \mathrm{CI})\end{array}$ & $\begin{array}{l}\text { Fully adjusted HR } \\
(95 \% \mathrm{CI})\end{array}$ & $\begin{array}{l}\text { Unadjusted HR } \\
(95 \% \mathrm{CI})\end{array}$ & $\begin{array}{l}\text { Fully adjusted } \mathrm{HR} \\
(95 \% \mathrm{CI})\end{array}$ \\
\hline No morbidity & 0.45 & 1.00 & 1.00 & 1.00 & 1.00 & 1.00 & 1.00 \\
\hline Single physical & 1.67 & $\begin{array}{l}3.74(3.53 \\
3.96)^{* * *}\end{array}$ & $\begin{array}{l}2.31(2.18 \\
2.45)^{* * *}\end{array}$ & $\begin{array}{l}3.33(3.09 \\
3.60) * * *\end{array}$ & $\begin{array}{l}2.07(1.91 \\
2.24)^{* * *}\end{array}$ & $\begin{array}{l}4.30(3.93 \\
4.71)^{* * *}\end{array}$ & $\begin{array}{l}2.71(2.47 \\
2.98)^{* * *}\end{array}$ \\
\hline Single mental/cognitive & 1.09 & $\begin{array}{l}2.44(2.19 \\
2.71)^{* * *}\end{array}$ & $\begin{array}{l}1.77(1.59 \\
1.97)^{* * *}\end{array}$ & $\begin{array}{l}2.93(2.56 \\
3.36)^{* * *}\end{array}$ & $\begin{array}{l}1.82(1.58 \\
2.09)^{* * *}\end{array}$ & $\begin{array}{l}2.10(1.78 \\
2.49)^{* * *}\end{array}$ & $\begin{array}{l}1.72(1.45 \\
2.04)^{* * *}\end{array}$ \\
\hline Multimorbid physical & 3.65 & $\begin{array}{l}8.25(7.79 \\
8.74)^{* * *}\end{array}$ & $\begin{array}{l}3.55(3.34 \\
3.78)^{* * *}\end{array}$ & $\begin{array}{l}7.56(7.01 \\
8.16)^{* * *}\end{array}$ & $\begin{array}{l}3.20(2.95 \\
3.47)^{* * *}\end{array}$ & $\begin{array}{l}9.34(8.55 \\
10.21)^{* * *}\end{array}$ & $\begin{array}{l}4.11(3.74 \\
4.51)^{* * *}\end{array}$ \\
\hline Multimorbid mental/cognitive & 2.06 & $\begin{array}{l}4.62(3.49 \\
6.13)^{* * *}\end{array}$ & $\begin{array}{l}2.62(1.97 \\
3.48)^{* * *}\end{array}$ & $\begin{array}{l}4.62(3.34 \\
6.40)^{* * *}\end{array}$ & $\begin{array}{l}2.52(1.81 \\
3.50)^{* * *}\end{array}$ & $\begin{array}{l}3.74(2.12 \\
6.61)^{* * *}\end{array}$ & $\begin{array}{l}2.49(1.41 \\
4.40)^{* * *}\end{array}$ \\
\hline $\begin{array}{l}\text { Multimorbid } \\
\text { physical-mental/cognitive }\end{array}$ & 3.19 & $\begin{array}{l}7.20(6.77 \\
7.66)^{* * *}\end{array}$ & $\begin{array}{l}3.32(3.10 \\
3.54)^{* * *}\end{array}$ & $\begin{array}{l}7.08(6.52 \\
7.69)^{* * *}\end{array}$ & $\begin{array}{l}2.98(2.73 \\
3.26)^{* * *}\end{array}$ & $\begin{array}{l}7.76(7.07 \\
8.52)^{* * *}\end{array}$ & $\begin{array}{l}3.84(3.47 \\
4.25)^{* * *}\end{array}$ \\
\hline
\end{tabular}

Results represent HR and 95\% Cls from Cox Proportional Hazard models.

Models adjusted for all included indicators: sex (in model including 'all persons'), marital status, rateable value of property, household car access, educational attainment and area of residence.

${ }^{* * *} P<0.001$. 
The study also noted a negative gradient associated with multimorbidity and socio-economic circumstance, which was more marked among younger age groups. Our findings are consistent with increased risk of multimorbidity with age but suggest a stronger gradient between age and morbidity combinations that include physical conditions for both males and females. All three indicators of socio-economic circumstance were also consistently predictive of multimorbidity. Importantly, our study showed an elevated risk of morbidity for outcomes with a mental health component among the most socially deprived, echoing previous research in Scotland. ${ }^{5}$ In a review of evidence on the link between SEC and mental health, Muntaner et al. ${ }^{18}$ consider potential mechanisms underpinning this relationship, focusing on the causal direction of the link between SEC and depression and the effect of early life course influences among more deprived population groups on mental health problems in adulthood.

Beyond age, gender and SEC, little is known about other social characteristics associated with multimorbidity, particularly among younger adults. A population-based approach affords the opportunity to conduct a fine-grained analysis with respect to multimorbidity, exploring a wider range of social determinants than has been previously considered.

\section{What this study adds}

Our findings are derived from whole population Census data and include rich and robust socio-economic data on individual and household circumstance. The study strengthens the evidence base on the importance of examining multimorbidity in younger age groups. Additionally, few studies have been able to examine the combinations of disorders and their social determinants. Thus, while multimorbidity per se is consistently predictive of mortality, ${ }^{19-22}$ we found higher mortality risks associated with all three multimorbidity categories, with risk most elevated for profiles that included a physical condition. That said that the elevated risk of mortality associated with multimorbid mental/cognitive conditions also raises important questions for research and service planning. Higher mortality among multimorbid individuals may reflect complex mechanisms and interactions, including the association of multimorbidity with frailty, functional impairment and disability, ${ }^{20,21,23}$ the role of social support, ${ }^{22}$ and the impact of fragmented care. ${ }^{19}$ Further in-depth analysis on causespecific mortality and multimorbidity clusters, particularly among younger and middle-aged adults, would enhance the evidence base and inform the management and targeting of resources for combinations of multimorbid conditions.

As with other studies, ${ }^{24-26}$ we found females were more likely to report multimorbidity, per se. Further sex-specific analysis of profiles of morbidity and multimorbidity, however, suggest some differences between males and females in relation to other characteristics associated with different profiles of morbidity, which are discussed below.

Marriage is known to be protective against morbidity, ${ }^{27-28}$ and we confirmed that those widowed/separated or divorced had an elevated risk of multimorbidity per se and all profiles of morbidity. For both males and females, however, those never married were less likely to report multimorbid physical health outcomes, but more likely to report multimorbid outcomes with a mental/cognitive component. Further exploration of the association of marital status and the influence of lifestyle factors and social support/networks may be useful to untangle these complex associations.

People living in urban communities have been found to have higher risk of poorer physical and mental health and premature mortality. ${ }^{29-32}$ While urban-rural inequalities with respect to multimorbidity is less well evidenced, Garin $e t$ al. ${ }^{33}$ reported similar findings in a cross-national study. However, while we noted lower risk of physical multimorbidity among females resident in intermediate and rural settings, the same group had an increased risk of mental/cognitive multimorbidity, not found in other studies. ${ }^{30,32}$ While further research and potential refinement of multimorbid categories is required, urban/rural disparities may be explained by other factors including social environment, isolation, stigma, access to services, access to green spaces ${ }^{34}$ and experience of life events. $^{35}$

\section{Limitations of this study}

The estimates of multimorbidity were derived from Census self-reports. While evidence on the validity of self-reported chronic conditions obtained from Census data is limited, earlier validation studies of self-reported chronic conditions point to reasonable accuracy for a range of conditions ${ }^{36,37}$ and conclude that self-reports are valid for effective use in population health management interventions. ${ }^{37}$ Secondly, the broad nature of questions relating to the range of conditions made categorisation into morbidity categories (physical and mental/cognitive) and associated 'multimorbidity categories' problematic. Finally, it is likely that the prevalence of multimorbidity (particularly multimorbid mental/cognitive conditions) is underestimated, given the broad nature of the question: the question relating to mental health potentially encompasses a range of mood, anxiety and behavioural conditions.

\section{Implications and future research}

There are considerable benefits in using Census data to enhance basic knowledge of the prevalence and risk of multimorbidity and associated mortality in the general 
population. Further work is required in refining and validating census-based self-reported health indicators, particularly in relation to mental health. Our study affirms that multimorbidity poses a persisting challenge in Western populations, where multimorbidity and complex multimorbidity are projected to rise. ${ }^{38}$ While clinicians are aware of the association of multimorbidity, age and socio-economic circumstances, they must also recognise patterns of multimorbidity and risk factors in younger and middle-aged groups. Thus the evidence base on multimorbidity should include younger and middleaged adults and further studies are needed to explore the complex clustering of multiple conditions, risk factors and how these change across the lifespan.

\section{Acknowledgements}

The help provided by the staff of the Northern Ireland Mortality Study (NIMS) and the Northern Ireland Longitudinal Study Research Support Unit (NILS-RSU) is acknowledged. The NIMS is funded by the Health and Social Care Research and Development Division of the Public Health Agency (HSC R\&D Division) and Northern Ireland Statistics and Research Agency (NISRA). The NILS-RSU is funded by the Economic and Social Research Council (ESRC) and the Northern Ireland Government. The authors alone are responsible for the interpretation of the data and any views or opinions presented are solely those of the author and do not necessarily represent those of NISRA/NILS.

\section{Conflict of interest}

None declared.

\section{Funding}

This work was supported by the Economic and Social Research Council (ESRC) (project number: ES/L007509/1).

\section{References}

1 World Health Organisation. Multimorbidity: Technical Series on Safer Primary Care. Geneva: World Health Organization, 2016.

2 Nguyen H, Manolova G, Daskalopoulou C et al. Prevalence of multimorbidity in community settings: a systematic review and meta-analysis of observational studies. Journal of comorbidity 2019;9: $2235042 X 19870934$.

3 Allison F, Lix Lisa M, Kim R. Estimating multimorbidity prevalence with the Canadian chronic disease surveillance system. Health promotion and chronic disease prevention in Canada: research, policy and practice 2017;37(7):215.
4 de Carvalho JN, Roncalli ÂG, de Camargo Cancela M et al. Prevalence of multimorbidity in the Brazilian adult population according to socioeconomic and demographic characteristics. PloS one. 2017;12(4):e0174322.

5 Barnett K, Mercer SW, Norbury M et al. Epidemiology of multimorbidity and implications for health care, research, and medical education: a cross-sectional study. The Lancet. 2012;380(9836):37-43.

6 Puth MT, Weckbecker K, Schmid M et al. Prevalence of multimorbidity in Germany: impact of age and educational level in a cross-sectional study on 19,294 adults. BMC public health. 2017;17(1):826.

7 Prazeres F, Santiago L. Prevalence of multimorbidity in the adult population attending primary care in Portugal: a cross-sectional study. BMJ open. 2015;5(9):e009287.

8 Fortin M, Bravo G, Hudon C et al. Prevalence of multimorbidity among adults seen in family practice. The Annals of Family Medicine. 2005;3(3):223-8.

9 Picco L, Achilla E, Abdin E et al. Economic burden of multimorbidity among older adults: impact on healthcare and societal costs. BMC bealth services research. 2016;16(1):173.

10 Salive ME. Multimorbidity in older adults. Epidemiologic reviews. 2013;35(1):75-83.

11 de Cock TP, Rosato M, Ferry F et al. Patterns of long-term conditions in older age and subsequent mortality: a national study of inequalities in health. European journal of public bealth. 2019.

12 O'Reilly D, Rosato M, Catney G et al. Cohort description: the Northern Ireland longitudinal study (NILS). International Journal of Epidemiolog). 2011;41(3):634-41.

13 Navickas R, Petric VK, Feigl AB et al. Multimorbidity: what do we know? What should we do? Journal of comorbidity. 2016;6(1):4-11.

14 Mair FS, Gallacher KL. Multimorbidity: what next? $\mathrm{Br} J$ Gen Pract. 2017;67(659):248-9.

15 Taylor AW, Price K, Gill TK et al. Multimorbidity-not just an older person's issue. Results from an Australian biomedical study. BMC public bealth. 2010;10(1):718.

16 McLean G, Gunn J, Wyke S et al. The influence of socioeconomic deprivation on multimorbidity at different ages: a cross-sectional study. Br J Gen Pract. 2014;64(624):e440-7.

17 Violán C, Foguet-Boreu Q, Flores-Mateo G et al. Prevalence, determinants and patterns of multimorbidity in primary care: a systematic review of observational studies. PloS one. 2014;9(7):e102149.

18 Afshar S, Roderick PJ, Kowal P et al. Multimorbidity and the inequalities of global ageing: a cross-sectional study of 28 countries using the world health surveys. BMC Public Health. 2015;15(1):776.

19 Muntaner C, Eaton WW, Miech R et al. Socioeconomic position and major mental disorders. Epidemiologic reviews. 2004;26(1):53-62.

20 Nunes BP, Flores TR, Mielke GI et al. Multimorbidity and mortality in older adults: a systematic review and meta-analysis. Archives of gerontology and geriatrics. 2016;67:130-8.

21 Wei MY, Multimorbidity MKJ. Mortality, and long-term physical functioning in 3 prospective cohorts of community-dwelling adults. American journal of epidemiology. 2017;187(1):103-12.

22 St John PD, Tyas SL, Menec V et al. Multimorbidity, disability, and mortality in community-dwelling older adults. Canadian family physician. 2014;60(5):e272-80. 
23 Olaya B, Domènech-Abella J, Moneta MV et al. All-cause mortality and multimorbidity in older adults: the role of social support and loneliness. Experimental gerontology. 2017;99:120-6.

24 Nguyen QD, Wu C, Odden MC et al. Multimorbidity patterns, frailty, and survival in community-dwelling older adults. The Journals of Gerontology: Series A. 2019;74(8):1265-70.

25 Abad-Díez JM, Calderón-Larrañaga A, Poncel-Falcó A et al. Age and gender differences in the prevalence and patterns of multimorbidity in the older population. BMC geriatrics. 2014;14(1):75.

26 Gorman BK, Read JN. Gender disparities in adult health: an examination of three measures of morbidity. Journal of health and social behavior. 2006;47(2):95-110.

27 Tsang A, Von Korff M, Lee S et al. Common chronic pain conditions in developed and developing countries: gender and age differences and comorbidity with depression-anxiety disorders. The journal of pain. 2008;9(10):883-91.

28 Verbrugge LM. Marital status and health. Journal of Marriage and the Family. 1979;267-85.

29 Umberson D. Gender, marital status and the social control of health behavior. Social science \&o medicine. 1992;34(8):907-17.

30 Riva M, Curtis S, Gauvin L et al. Unravelling the extent of inequalities in health across urban and rural areas: evidence from a national sample in England. Social science \& medicine. 2009;68(4): 654-63.

31 Peen J, Schoevers RA, Beekman AT et al. The current status of urbanrural differences in psychiatric disorders. Acta Psychiatrica Scandinavica. 2010;121(2):84-93.
32 O'Reilly G, O'Reilly D, Rosato M et al. Urban and rural variations in morbidity and mortality in Northern Ireland. BMC Public Health. 2007;7(1):123.

33 Kovess-Masféty V, Alonso J, de Graaf R et al. A European approach to rural-urban differences in mental health: the ESEMeD 2000 comparative study. The Canadian Journal of Psychiatry. 2005;50(14):92636.

34 Garin N, Koyanagi A, Chatterji S et al. Global multimorbidity patterns: a cross-sectional, population-based, multi-country study. Journals of gerontology series a: biomedical sciences and medical. Sciences. 2016;71(2):205-14.

35 Mitchell R, Popham F. Effect of exposure to natural environment on health inequalities: an observational population study. The Lancet. 2008;372(9650):1655-60.

36 Prudo R, Brown GW, Harris T et al. Psychiatric disorder in a rural and an urban population: 2. Sensitivity to loss. Psychological Medicine. 1981;11(3):601-16.

37 Lee Y. The predictive value of self assessed general, physical, and mental health on functional decline and mortality in older adults. Journal of Epidemiology \& Community Health. 2000;54(2):123-9.

38 Chapman, L. How valid is self-reported health data? A Chapman Institute white paper. Chapman Institute. 2012. https://chapmaninstitute.com/resou rces/valid-self-reported-health-data/. Accessed February 2018.

39 Kingston A, Robinson L, Booth $\mathrm{H}$ et al. MODEM project. Projections of multi-morbidity in the older population in England to 2035: estimates from the population ageing and care simulation (PACSim) model. Age and ageing. 2018;47(3):374-80. 